\title{
Peculiarity of using the paleotsunami data for the tsunami hazard estimation
}

\author{
Victor M. Kaistrenko
}

\begin{abstract}
Institute of Marine Geology and Geophysics FEB RAS, Yuzhno-Sakhalinsk, Russia
\end{abstract}

*E-mail:vict-k@mail.ru

\begin{abstract}
Peфepat PDF Rus
The work is focused on the developing of the methods of the tsunami recurrence evaluation using paleotsunami data. The new key moment is the model for the quantitative evaluation of the preservation potential of the paleotsunami deposits. The short topical review on the preservation / changeability of the paleotsunami deposits is included. Paleotsunami data found earlier on the Khalaktyrka coast near Petropavlovsk-Kamchatskiy have been used for testing of the developed model. All the data have been divided and considered separately according to the time intervals between marker explosions of volcanoes Ksudach (1907), Avachinskiy (1855 and 1779) and Opala (606). The maximal confident estimates of the tsunami deposit numbers have been received as well as their probability of preservation. Some restrictions related to the model conditions have been analyzed.
\end{abstract}

\section{Keywords}

tsunami, run-up height, paleotsunami, tsunami deposits, recurrence, tsunami hazard, probabilistic model, statistics

For citation: Kaistrenko V.M. Peculiarity of using the paleotsunami data for the tsunami hazard estimation. Geosystems of Transition Zones. 2019, vol. 3, no. 4, pp. 403-416. (In Russian) https://doi.org/10.30730/25418912.2019.3.4.403-416

Для цитирования: Кайстренко В.М. Особенности использования данных о палеоцунами для оценок цунамиопасности. Геосистемы переходных зон. 2019. Т. 3, № 4. С. 403-416. https://doi.org/10.30730/25418912.2019.3.4.403-416

\section{References}

1. Бобыкина В.П. Цунами как экзогенный берегоформирующий фактор // Новые данные по геоморфологии и геологии западной части Тихого океана. Владивосток: ТОИ ДВО АН СССР, 1990. С. 51-57.

2. Булгаков Р.Ф., Иванов В.В., Певзнер М.М., Сулержицкий Л.Д., Храмушин В.Н. Исследование следов палеоцунами для цунамирайонирования // Физика Земли. 1995. № 2. С. 18-27.

3. Ганзей Л.А., Разжигаева Н.Г., Нишимура Ю., Гребенникова Т.А., Кайстренко В.М., Горбунов А.О., Арсланов Х.А., Чернов С.Б. Наумов Ю.А. Осадки исторических и палеоцунами на побережье Восточного Приморья // Тихоокеанская геология. 2015. № 1. С. 79-95. [Ganzey L.A., Razjigaeva N.G., Nishimura Yu., Grebennikova T.A., Kaistrenko V.M., Gorbunov A.O., Arslanov Kh.A., Chernov S.B., Naumov Yu.A. Deposits of historical and paleotsunamis on the coast of Eastern Primorye // Russian J. of Pacific Geology. 2015, 9(1): 64-79. doi.org/10.1134/S1819714015010029]

4. Ганзей Л.А., Разжигаева Н.Г., Нишимура Ю., Гребенникова Т.А., Горбунов А.О., Кайстренко В.М., Наумов Ю.А., Лебедев И.И. Осадки цунами 1983 и 1993 годов на побережье Приморья // Океанология. 2017. T. 57(4). C. 628-640 [Ganzey L.A., Razjigaeva N.G., Nishimura Yu., Grebennikova T.A., Gorbunov A.O., Kaistrenko V.M., Naumov Yu.A., Lebedev I.I. Deposits of the 1983 and 1993 tsunamis on the coast of Primorye // Oceanology. 2017, 57(4): 568-579. https://doi.org/10.1134/S0001437017040075] 
5. Иванов В.В. Исследование влияния цунами на осадконакопление по следам цунами 4 октября 1994 года // Проявления конкретных иунами. Цунами 1993 и 1994 годов на побережье России. Южно-Сахалинск: ИМГиГ ДВО РАН, 1997. С. 119-128. (Геодинамика тектоносферы зоны сочленения Тихого океана с Евразией; т. 8).

6. Кайстренко В.М., Клячко М.А., Храмушин В.Н., Золотухин Д.Е. Оценка цунамиопасности и построение карт цунамирайонирования для побережья Сахалинской области. Проблема нормативов // Строительная индустрия Сахалина. 2014. № 19. С. 38-43.

7. Каплин П.А., Ионин А.С. Некоторые особенности рельефа побережья Курило-Камчатской зоны в связи с проблемой цунами // Бюл. Совета по сейсмологии АН СССР. 1961. № 9. С. 74-88.

8. Крамер Г. Математические методы статистики. М.: Мир, 1975. 648 с. [Cramer H. Mathematical Methods of Statistics. Bombay; New-York: Asia Publ. House, 1946. 575 p.]

9. Лаврентьев М.А., Шабат Б.В. Методы теории функиий комплексного переменного. М.: Наука, 1987. $688 \mathrm{c}$.

10.Левин Б.В., Носов М.А. Физика иунами и родственных явлений в океане. М.: Янус-К, 2005. 360 с. [Levin B.W., Nosov M.A. Physics of Tsunamis. 2nd ed. Springer Intern. Publ. Switzerland, 2016. 388 p. https://doi.org/10.1007/978-3-319-24037-4]

11.Пинегина Т.К., Буржуа Д., Базанова Л.И., Брайцева О.А., Егоров Ю.О. Отложения цунами и анализ цунамиопасности на Халактырском пляже в районе Петропавловска-Камчатского, Тихоокеанское побережье Камчатки, Россия // Сб. статей по результатам междунар. конф. «Локальные иунами: предупреждение и уменьшение риска», Петропавловск-Камчатский, 10-15 сентября 2002 г. М.: Янус-К, 2002. C. 142-151. [Pinegina T.K., Bourgeois J., Bazanova L.I., Braitseva O.A., Egorov Yu.O. Tsunami and analysis of tsunami risk at Khalaktyrka beach region of Petropavlovsk-Kamchatsky, Pacific coast of Kamchatka. In: Proceedings of the International workshop "Local tsunami warning and mitigation", Petropavlovsk-Kamchatsky, September 10-15, 2002. English book. Moskow: Ianus-K, 2002. P. 122-131.]

12.Пинегина Т.К., Разжигаева Н.Г. Исследования палеоцунами на дальневосточном побережье России // Мировой океан. T. І. Геология и тектоника океана. Катастрофические явления в океане. М.: Науч. мир, 2013. С. 488-498.

13.Разжигаева Н.Г., Ганзей Л.А., Гребенникова Т.А., Харламов А.А., Ильев А.Я. Кайстренко В.М. Осадки цунами Шикотанского землетрясения 1994 г. // Океанология. 2007. Т. 47(4). С. 622-630. [Razjigaeva N.G., Ganzey L.A., Grebennikova T.A., Kharlamov A.A., Iliev A.Ya., Kaistrenko V.M. Tsunami Deposits of the Shikotan Earthquake of 1994 // Oceanology. 2007, 47(4): 579-587.

https://doi.org/10.1134/S0001437007040157]

14.Разжигаева Н.Г., Ганзей Л.А., Гребенникова Т.А., Харламов А.А., Ильев А.Я., Кайстренко В.М. Геологическая летопись палеоцунами на о. Шикотан (Малая Курильская гряда) в голоцене // Вулканология и сейсмология. 2008. № 4. C. 50-66. [Razzhigaeva N.G., Ganzei L.A., Grebennikova T.A., Kharlamov A.A., Il'ev A.Ya., Kaistrenko V.M. The geological record of paleotsunamis striking Shikotan Island, in the Lesser Kurils, during Holocene time // J. of Volcanology and Seismology. 2008, 2(4): 262-277. https://doi.org/10.1134/S0742046308040040]

15.Разжигаева Н.Г., Ганзей Л.А., Гребенникова Т.А., Харламов А.А., Кайстренко В.М., Арсланов Х.А., Горбунов А.О. Проявление палеоцунами на Малой Курильской гряде в голоцене // Тихоокеанская геология. 2012. T. 31, № 6. C. 48-56 [Razzhigaeva N.G., Ganzei L.A., Grebennikova T.A., Kharlamov A.A., Kaistrenko V.M., Arslanov Kh.A., Gorbunov A.O. Manifestation of Holocene Tsunamis on the Lesser Kuril Ridge // Russian J. of Pacific Geology. 2012, 6(6): 448-456. https://doi.org/10.1134/S1819714012060061]

16.Свод правил «Здания и сооружения в иунамиопасных районах. Правила проектирования» (СП 292.1325800.2017) / Клячко М.А., Зайцев А.И., Заритовский Н.Г., Кайстренко В.М., Максимов В.В., Нуднер И.С., Пелиновский Е.Н., Рутман Ю.Л., Фильков В.Ю., Штанько Л.Ф. М.: Минстрой России, 2017. $138 \mathrm{c}$.

17.Abe T., Goto K., Sugawara D. Relationship between the maximum extent of tsunami sand and the inundation limit of the 2011 Tohoku-oki tsunami on the Sendai Plain, Japan // Sedimentary Geology. 2012. Vol. 282. P. 142-150. https://doi.org/10.1016/j.sedgeo.2012.05.004

18. Atwater B.F., Moore A.L. A tsunami about 1000 years ago in Puget Sound, Washington // Science. 1992. Vol. 258(5088). P. 1614-1617. doi:10.1126/science.258.5088.1614

19.Bourgeois J., Hansen T.A, Wiberg P.L., Kauffman E.J. A tsunami deposit at the Cretaceous-Tertiary boundary in Texas // Science. 1988. Vol. 241(4865). P. 567-570. doi:10.1126/science.241.4865.567

20.Bourgeois J., Pinegina T.K., Ponomareva V., Zaretskaia N. Holocene tsunamis in the southwestern Bering Sea, Russian Far East, and their tectonic implications // GSA Bulletin. 2006. Vol. 118(3/4). P. 449-463. https://doi.org/10.1130/B25726.1

21.Braitseva O.A., Ponomareva V.V., Sulerzitsky L.D., Melekestsev I.V., Bailey J. Holocene key-marker tephra layers in Kamchatka, Russia // Quaternary Research. 1997. Vol. 47. P. 125-139.

https://doi.org/10.1006/qres.1996.1876

22.Chagué-Goff C., Goff J., Wong H.K.Y., Cisternas M. Insights from geochemistry and diatoms to characterise a tsunami's deposit and maximum inundation limit // Marine Geology. 2015. Vol. 359. P. 22-34. 
23. Cheng W., Weiss R. On sediment extent and run-up of tsunami waves // Earth and Planetary Science Letters. 2013. Vol. 362. P. 305-309. https://doi.org/10.1016/j.eps1.2012.12.004

24.Dawson A.G., Long D., Smith D.E. The Storegga slides: evidence from eastern Scotland for a possible tsunami // Marine Geology. 1988. Vol. 82. P. 271-276. https://doi.org/10.1016/0025-3227(88)90146-6

25.Einsele G., Chough S.K., Shiki T. Depositional events and their records - an introduction // Sedimentary Geology. 1996. Vol. 104. P. 1-9. https://doi.org/10.1016/0037-0738(95)00117-4

26.Geist E.L., Parsons T. Distribution of tsunami interevent times // Geophysical Research Letters. 2008. Vol. 35, L02612. https://doi.org/10.1029/2007GL032690

27.HTDB/WLD: Historical Tsunami Database for the World Ocean. URL: http://tsun.sscc.ru/htdbpac/ (accessed: 15.09.2014).

28.Jaffe A.E., Gelfenbaum G. A simple model for calculating tsunami flow speed from tsunami deposits // Sedimentary Geology. 2007. Vol. 200(3-4). P. 347-361. https://doi.org/10.1016/j.sedgeo.2007.01.013

29. Kaistrenko V. Tsunami recurrence versus tsunami height distribution along the coast // Pure and Applied Geophysics. 2011. Vol. 168(11). P. 2065-2069. https://doi.org/10.1007/s00024-011-0288-0

30. Kaistrenko V. Tsunami recurrence function: Structure, methods of creation, and application for tsunami hazard estimates // Pure and Applied Geophysics. 2014. Vol. 171. P. 3527-3538. https://doi.org/10.1007/s00024-0140791-1

31.Kaistrenko V.M., Pinegina T.K., Klyachko M.A. Evaluation of tsunami hazard for the Southern Kamchatka coast using historical and paleotsunami data // Submarine Landslides and Tsunamis: Proceedings of the NATO Advanced Research Workshop, Istanbul, Turkey, 23-26 May 2001 / eds.: A.C. Yalciner, E. Pelinovsky, C.E. Synolakis, E. Okal. Kluwer Publ., Springer, 2003. P. 217-228.

32.Kastens K.A., Cita M.B. Tsunami-induced sediment transport in the abyssal Mediterranean Sea // GSA Bulletin. 1981. Vol. 92(11). P. 845-857. https://doi.org/10.1130/0016-7606(1981)92<845:TSTITA>2.0.CO;2

33. Keating B.H. Status of tsunami science research and future directions of research // Science of Tsunami Hazards. 2006. Vol. 24(5). P. 285-395.

34.Keating B.H., Helsley C.E., Wanink M., Walker D. Tsunami deposit research: Fidelity of the tsunami record, ephemeral nature, tsunami deposits characteristics, remobilization of sediment by later waves, and boulder movements // The Tsunami Threat - Research and Technology / ed. by Nils-Axel Mörner. Rijeka, Croatia: InTech, 2011. P. 389-422. https://doi.org/10.5772/14885

35.MacInnes B.T., Bourgeois J., Pinegina T.K., Kravchunovskaya E.A. Tsunami geomorphology: Erosion and deposition from the 15 November 2006 Kuril Island tsunami // Geology. 2009a. Vol. 37(11). P. 995-998. doi.org/10.1130/G30172A.1

36.MacInnes B.T., Pinegina T.K., Bourgeois J., Razzhigaeva N.G., Kaistrenko V.M., Kravchunovskaya E.A. Field survey and geological effects of the 15 November 2006 Kuril tsunami in the middle Kuril Islands // Pure and Applied Geophysics. 2009b. Vol. 166(1-2). P. 3-36 https://doi.org/10.1007/978-3-0346-0064-4_2

37. Marinatos S. The volcanic destruction of Minoan Crete // Antiquity. 1939. Vol. 13. P. 425-439.

38. Minoura K., Nakata T. Discovery of an ancient tsunami deposit in coastal sequences of southwest Japan: verification of a large historical tsunami // Island Arc. 1994. Vol. 3. P. 66-72. https://doi.org/10.1111/j.1440$\underline{1738.1994 . t b 00006 . x}$

39. Minoura K., Nakaya S. Traces of tsunami preserved in inter-tidal lacustrine and marsh deposits: some examples from northeast Japan // The Journal of Geology. 1991. Vol. 99. P. 265-287.

40.Moore G.W., Moore J.G. Deposits from a giant wave on the island of Lanai, Hawaii // Science. 1984. Vol. 226. P. 1312-1315. doi:10.1126/science.226.4680.1312

41.Moor J.G., Moore G.W. Large-scale bedforms in boulder gravel produced by giant waves in Hawaii // Geological Society of America Special. 1986. Vol. 229. P. 101-110. https://doi.org/10.1130/SPE229-p101

42.NGDC: Tsunami Data and Information. URL: https://www.ngdc.noaa.gov/hazard/tsu_db.shtml (accessed: 29.09.2019). doi:10.7289/V5PN93H7

43. Oliveira M.A., Andrade C., Freitas M.C., Costa P. Using the historical record and geomorphological setting to identify tsunami deposits in the southwestern coast of Algarve (Portugal) // 2nd Tsunami Field Symposium: Abstract Book. Ostuni, Italy, 2008. P. 85-88.

44.Paskoff R. Likely occurrence of a mega-tsunami in the Middle Pleistocene, near Coquimbo, Chile // Revista Geológica de Chile. 1991. Vol. 18(1). P. 87-91. doi:10.5027/andgeoV18n1-a08

45.Pinegina T., Bourgeois J., Bazanova L., Melekestsev I., Braitseva O.A. Millennial - scale record of Holocene tsunamis on the Kronotskiy Bay coast, Kamchatka, Russia // Quaternary Research. 2003. Vol. 59. P. 36-47. https://doi.org/10.1016/S0033-5894(02)00009-1

46.Razjigaeva N.G., Ganzey L.A., Grebennikova T.A., Ivanova E.D., Kharlamov A.A., Kaistrenko V.M., Shishkin A.A. Coastal sedimentation associated with the Tohoku Tsunami of 11 March 2011 in South Kuril Islands, NW Pacific Ocean // Pure and Applied Geophysics. 2013. Vol. 170(6-8). P. 1081-1102. https://doi.org/10.1007/s00024-012-0478-4

47.Razjigaeva N.G., Ganzey L.A., Grebennikova T.A., Ivanova E.D., Kharlamov A.A., Kaistrenko V.M., Arslanov Kh.A., Chernov S.B. The Tohoku Tsunami of 11 March 2011: The key event to understanding 
tsunami sedimentation on the coasts of closed bays of the Lesser Kuril Islands // Pure and Applied Geophysics. 2014. Vol. 171(12). P. 3307-3320. https://doi.org/10.1007/s00024-014-0794-y

48. Soulsby R., Smith D.E., Ruffman A. Reconstructing tsunami run-up from sedimentary characteristics: A simple mathematical model // Sixth Intern. Symposium on Coastal Engineering and Science of Coastal Sediment Process, New Orleans, Louisiana, United States, May 13-17, 2007. Coastal Sediments '07. New Orleans, 2007. P. 1075-1088.

49.Spiske M., Piepenbreier J., Benavente C., Bahlburg H. Preservation potential of tsunami deposits on arid siliciclastic coasts // Earth Science Reviews. 2013. Vol. 126. P. 58-73.

http://dx.doi.org/10.1016/j.earscirev.2013.07.009

50. Srisutam Ch., Wagner J-F. Reconstructing tsunami run-up from the characteristics of tsunami deposits on the Thai Andaman Coast // Coastal Engineering. 2010. Vol. 57. P. 493-499. https://doi.org/10.1016/j.coastaleng.2009.12.001

51.Szczucin'ski W. The post-depositional changes of the onshore 2004 tsunami deposits on the Andaman Sea coast of Thailand // Natural Hazards. 2012. Vol. 60. P. 115-133. https://doi.org/10.1007/s11069-011-9956-8

52.Witter R.C., Jaffe B., Zhang Y., Priest G. Reconstructing hydrodynamic flow parameters of the 1700 tsunami at Cannon Beach, Oregon, USA // Natural Hazards. 2012. Vol. 63. P. 223-240. https://doi.org/10.1007/s11069$\underline{011-9912-7}$ 